\title{
Hybrid Multi-Objectives Genetic Algorithms and Immigrants Scheme for Dynamic Routing Problems in Mobile Networks
}

\author{
Khalil Ibrahim Mohammad Abuzanouneh \\ IT Department, College of Computer \\ Qassim University \\ Al Qassim, Saudi Arabia
}

\begin{abstract}
In this paper, the author discusses the main concept of intelligent optimization techniques, artificial neural networks, and new genetic algorithms to solve the multi-objective multicast routing problems with shortest path (SP) problem that used in the addresses networks and improve all processes addressing in the wireless communications based on multiobjective optimization. The most important characteristics in mobile wireless networks is the topology dynamics and the network topology changes over time, the routing problem (SPRP) in mobile ad hoc networks (MANETs) turns out to be a dynamic optimization problem[13], the hybrid immigrants multiple-objective genetic algorithm (HIMOGAs) in the realworld are dynamic in nature, that has objective functions, constraints, and parameters, the dynamic optimization problems (DOPs) are a big challenges to evolutionary multiobjective, since any environmental change may affect the objective vector, constraints, and parameters, HIMOGA for the optimization goal is to track the moving of parameters and get a sequence of approximations solutions over time. The quantity of services (QoS) is supporting guarantee for all data traffic and getting the maximizing utilization for network, the QoS based on multicast routing offer significant challenges, and increases to use an efficient multicast routing protocol that will be able to check multicast routing and satisfying QoS constraints, The author propose to use HIMOGAs and SP algorithm to solve multicast problem that produces new generation wireless networks with immigrants schema to get high-quality solutions after each change and satisfying all objectives.
\end{abstract}

\section{General Terms}

Genetic algorithms, Dynamic optimization problem, multipleobjectives algorithm

\section{Keywords}

Hybrid immigrants multiple-objective, dynamic shortest path routing problem, Dynamic immigrants scheme.

\section{INTRODUCTION}

Mobile ad hoc networks is a type of that can change locations and a continuously self-configuring, they use wireless connections to connect to various networks Such as Wi-Fi connection or satellite transmission, the dynamic nature of MANETs are typically not very secure and it is important to send data over a MANET[1], the main two types of the deterministic algorithms for the multicast problem: the deterministic algorithms and the search heuristics The deterministic algorithm such as the shortest path[2] tree (SPT) algorithm used to constructed multicast tree for any topology[3].

The search heuristics, such as genetic algorithms (GAs) is the best one of multicast trees are searched as the final result [4]. All the deterministic algorithms have polynomial time complexity and effective in fixed infrastructure wireless or wired networks, the search heuristics are worthy of investigation for high computational complexity in the realtime communications involving changing network topologies [7].

Studying evolutionary algorithms (EAs) for DOPs has a growing interest due to its importance in EA's real world applications [4]. The addressing DOPs is to restart EAs from the beginning whenever an environment change is updated. Ad ho networks have robust and efficient operations by using the routing functionality with mobile hosts. The unicast routing establishes a multi-hop forwarding path for two nodes beyond the direct

Wireless communication range [7]. The addressing DOPs need to restart EAs from the beginning whenever an environment change is updated. The restart scheme really works is more efficient to develop new immigrants scheme for knowledge gathering information from previous environments. during the run new immigrants [15],[16], to get more diversity after each change environment [8],used routing table scheme to reuse stored new data table [4], used applying multi-population and different schemes to search in different area of the search space[6], and updating the parameters of operators to get fast respond to a new environment[9].

In this paper, this research propose to use multi-objective the algorithm in MANET to define all parameters within the framework of the problem to get a better definition of the problem definition, and in turn facilitates access appropriate the solution for all parties. From here began pluralistic definition of goals and how to reach a solution that suits conflicting objectives to define the problem [12], the author had set goals must be achieved and taken into consideration when finding suitable solutions, so during the search for a solution to achieve these objectives to get a satisfactory solution for QoS constraints [18].

Routing protocols also maintain connectivity when links on these paths break, due to effects such as node movement, wireless interference or lost connection, there are two types of routing protocols in MANETs, topological routing, and geographic routing. In the topological routing, mobile nodes utilize the topological information to construct routing tables or search routes directly [14]. In the geographic routing, based on the position of the destination and the positions of its local neighbors, each node knows its own position and makes routing decisions.

The author propose and investigate the hybrid immigrants multiple-objectives genetic algorithms (HIMOGAs) with the 
shortest path algorithm [2] to solve routing a problem in the dynamic environments, which belongs to the topological routing, and QoS requirements with different network applications in collaborative group communications, such as the video conference service, which is the delivery of information simultaneously, used efficient strategy for distant education and content distribution. the real-time delivery of multimedia applications are required[21],[22], QoS the multicast algorithm should construct a multicast routing tree, for the data transmitted from the source to the destinations with a guaranteed and efficiently, the utilization of network resources are evaluated used the multicast tree cost.

In this research, the author was studied and investigated different genetic algorithms (GAs) that are developed and used to work with the real DOPs to solve the DSPRP in MANETs. First, this research designs all parameters of the genetic algorithm specifically for the DSPRP, and integrate several immigrants of memory schemes and their combination into the GA to enhance its searching capacity for the SPs in dynamic environments. Once the topology is changed, new immigrants or the useful information stored in the memory can help guide the search for good solutions in the new environment. For comparison purposes, and also implement two traditional GA schemes, i.e., SGA and restart GA, as the basic main algorithms. Via simulation experiments, the experiments evaluated these GAs under different parameter settings to find the best combinations.

The experimental results show that HIMOGAs can quickly adapt to the environmental changes and resources for increasing high-quality solutions with QoS requirements in different network applications and communications, the various algorithms that are developed to work with DOPs in the dynamic environments to evaluate their performance and solve the dynamic multicast routing problem were applied in the final experiments, the immigrant's scheme was organized and coordinated in the GA to improve its searching capacity in dynamic environments, this research implements traditional GA schemes, multiple objective genetic algorithms (MOGAs), random immigrants multiple objective genetic algorithms (RIMOGAs)[12], elitism immigrants multiple objective genetic algorithms (EIMOGAs)[15],[16] , and hybrid immigrants multiple objective genetic algorithms (HIMOGAs), By comparison, and simulation experiments, these GAs are evaluated under diff erent parameter settings to find the best combinations. In the dynamic environments applied different parameters and settings to evaluate the performance and find the best corresponding between algorithms and dynamic environmental characteristics[17].In Section II the related work is discussed. The DSPRP model is described in Section III. Section IV presents the design of a specialized GA for the static SP routing problem. The GAs that are the integration of several immigrants and/or memory schemes and the specialized GA for the DSPRP are described in Section V. The extensive experimental study and relevant analysis is presented in Section VI. Finally, Section VII concludes this paper with some discussions on the future work.

\section{RELATED WORK}

Deterministic algorithms produce multicast routing trees by using (SPT) [14]. SPT use to find the shortest minimum cost or delay path between the source and its destination and supply us a good solution to evaluate delay-constrained multicast tree with the minimum delay path from the source to each destination [11].
The heuristic algorithms are an intelligent search and good techniques to solve combinatorial optimization problems [8] applying the SPT problem, intelligent search heuristics is a type of promising techniques to solve combinatorial optimization problems including the SPT problem, and GAs are one of representative intelligent global search heuristics and one of stochastic meta-heuristic optimization methods ,GAs have been applied to solve the QoS for multicast problems in different networks such as the wired multimedia networks and optical networking system[21],[22], The multicast algorithms based on GAs that represents the binary encoding to adopt each bit of the binary string corresponds to a different node in the network[28], together, the binary string has a graph $G^{\prime}$ is derived from the network topology $G$ to include all the nodes in the network by appearing in the string and the links connecting together, the minimum tree $T$ of $\mathrm{G}^{\prime}$ considers the candidate multicast tree represented by the binary string, the encoding method is a complicated and each binary string cannot directly represent a good solution. The union of all routing paths is represented a multicast tree from the source to each destination for adopting the path-oriented encoding method.

The past few years in the MANETs, they are a several of multicast routing protocols have been proposed and using a different types of basic routing algorithms and techniques[14], the main focus on the research of the optimal multicast forwarding structure spanning mobile nodes and do not consider the last changes in the network topologies, the wireless mobile networks are a topology dynamics and inherent characteristics of its environment changing [10], where at time T1, the network topology is G1, at time T2, the network topology may change to G2. Although G1 and G2 are different, they are closely connected since each change alters segment of the network topology only, the candidate solutions into G1 could exploit the search of good solutions on G2. The multicast algorithm should track the topological changes and adapt the multicast trees.

\section{NETWORK MODEL AND DYNAMIC SP ROUTING PROBLEM}

In this section, the author first presents network model and then formulate the DSPRP to consider a MANET operating within a fixed geographical region. The model built by an undirected and connected topology graph $\mathrm{G0}(\mathrm{V} 0, \mathrm{E} 0)$, where V0 represents the set of wireless nodes and E0 represents the set of communication links connecting two neighboring routers that fall within the scope of broadcasting, a communication link (i, j) cannot be used for packet transmission unless both nodes $\mathrm{i}$ and node $\mathrm{j}$ have a radio interface and the message transmission to the wireless communications link incurred significantly delayed and the cost to summarize some of the symbols that used in this research: $\mathrm{G} 0(\mathrm{~V} 0, \mathrm{E} 0)$ the initial wireless topology graph; $\mathrm{Gi}(\mathrm{Vi}, \mathrm{Ei})$ wireless topology graph after i change; s source node; $r$ destination node; $\mathrm{Pi}(\mathrm{s}, \mathrm{r})$ path from $\mathrm{s}$ to $\mathrm{r}$ on the graph $\mathrm{Gi}$; Di transmission delay on the communication link $\mathrm{L}$; $\mathrm{Cl}$ cost on the communication link $\mathrm{L} ; \Delta(\mathrm{Pi})$ total transmission delay on the path $\mathrm{Pi} ; \mathrm{C}(\mathrm{Pi})$ the total cost of the path $\mathrm{Pi}$.

The DSPRP can be described as follows: a network of wireless routers, a delay, a source and a destination node, and to find the delay bounded least cost loop-free path on the topology graph, due to energy conservation or other problems, some sleeping nodes are scheduled to wake up, some waking up nodes are scheduled to sleep, and others network topology changes from time to time. The main objective of the DSPR P is to find the new optimal delay constrained least cost a cyclic 
path after each changing, from the source node to the destination node with the delay upper bound $\Delta$.

All links are associated with link cost $\mathrm{C}(\mathrm{e})$, bandwidth $\mathrm{B}(\mathrm{e})$ and delay $\mathrm{D}(\mathrm{e})$ respectively. A multicast tree is defined by $\mathrm{T}=$ $(\mathrm{VT}, \mathrm{ET})$, where $\mathrm{VT} \subseteq \mathrm{V}, \mathrm{ET} \subseteq \mathrm{E}$ and $\mathrm{G} \subseteq \mathrm{T}$. There exists a path PT(s, di) from the source node to each destination node Di $\in \mathrm{M}=\{\mathrm{d} 1 \ldots \mathrm{dk}\}$ in $\mathrm{T}$. where $\mathrm{k}$ is the number of destinations.

The research defined the multi-objective optimization problem that uses to find a multicast tree with minimizing the following four objectives [8]:

The delay sum of all links along PT(s, di) equals the total delay of the path PT (s, e) is.

$D\left(P_{T}(s, d)\right)=\sum_{e \epsilon P_{T}\left(s, d_{i}\right)}(D(e)$

The bandwidth of the path $P_{T}(\mathrm{~s}$, di) can be defined as the minimum available bandwidth at any the path link.

$\mathrm{B}\left(P_{T}(\mathrm{~s}, \mathrm{di})\right)=\min \left\{\mathrm{B}(\mathrm{e}), \mathrm{e} \in P_{T}(\mathrm{~s}, \mathrm{di})\right\}$

The Power constraint has defined the summation of the residual battery energy that the sender needs for a packet to the receiver.

$\operatorname{Pw}(\operatorname{PT}(\mathrm{s}, \mathrm{di}))=\min \{$ power( $(\mathrm{di})\}, \forall \mathrm{di} \in \mathrm{M}$

The total cost of the multicast tree can be defined as the cost sum of all links in the tree.

$\mathrm{C}(\mathrm{T}(\mathrm{s}, \mathrm{di}))=\sum_{e \in P_{T}\left(s, d_{i}\right)}(C(e)$

Using the previous definitions the considered problem can be expressed as follows: one a source node $\mathrm{s}$ link with a set of destination nodes $\mathrm{M}$, a delay tolerance Bbound and a bandwidth tolerance Bbound. To find a tree $\mathrm{T}(\mathrm{G} \subseteq \mathrm{T}$ ) routed at $\mathrm{s}$ and spanning all of the nodes in $\mathrm{M}$ such that the total cost of the multicast tree given in Eq. 7 is minimized and satisfied the QoS constraints.

The author used an energy-efficient localized topology control algorithm, to reduce the transmission power of the network nodes, extend the duration of the network lifetime simultaneously [20], and avoid overusing those links between energy-critical nodes to undertake the task of relaying packets. Let euv represent the minimal power required for a node $u$ to successfully transmit an information unit to another node $\mathrm{v}$, the power consumed transmission euv between two modes can be calculated as follows.

$$
\text { euv }=\mathrm{d}(\mathrm{u}, \mathrm{v}) \alpha+\mathrm{q}
$$

Where $\alpha$ and $\mathrm{q}$ stand for specific wireless systems, c stands for an energy-efficient (maximum signal max(s) processing and minimal energy min (e) required for successful reception $\}$. In (1), the interference model is adopted, in which the interference level is independent of network traffic and the same at all nodes. Moreover, e $s \rightarrow r$ is set to be infinite $(e s \rightarrow r$ $=\infty)$ if $\mathrm{d}(\mathrm{s}, \mathrm{r})>\mathrm{R}, \mathrm{R}$ represents the maximum uniform transmission range of nodes in the network. For a given $\mathrm{Ti}(\mathrm{s} \rightarrow \mathrm{r})=$ Path $\mathrm{P}$, connecting a pair of nodes, the overall power consumed for sending an information unit along $\mathrm{Ti}=\{\mathrm{s}, \mathrm{r} 1, \mathrm{r} 2, \mathrm{r} 3, .$.$\} is the sum of the link powers of its multicast$ tree $\mathrm{Ti}(\mathrm{s} \rightarrow \mathrm{r})$ as follows[20].

$$
e\left(P_{T}\right)=\sum_{e \in P_{T}(\mathrm{~s}, \mathrm{r})} \quad e(\mathrm{~s}, \mathrm{r})
$$

The minimum bandwidth ,the maximum delay, constraint of multicast tree are given a multicast demand $R=\left\{B_{\min }, D_{\max }\right\}$, the problem of multicast routing is to find a multicast tree $\mathrm{T}$, and $\mathrm{S}(\mathrm{R})$ is the set of tree satisfies all Bandwidth constraint , Delay constraint, Power constraint given in Eq. 9, means the summation of the residual battery energy that the sender needs for a packet to the receiver. The total delay (TD) and residual battery energy (RBE) functions associated with multicast tree given in Eq. 9 are described as:

$$
\begin{aligned}
& \mathrm{D}\left(P_{T}(\mathrm{~s}, \mathrm{di})\right) \leq \text { Dbound }=\max (\mathrm{D}(\mathrm{e})), \forall \mathrm{e} \in \mathrm{MT} \\
& \mathrm{B}\left(P_{T}(\mathrm{~s}, \mathrm{di})\right) \leq \mathrm{Bbound}=\min (\mathrm{B}(\mathrm{e})), \forall \mathrm{e} \in \mathrm{MT} \\
& \mathrm{J}(\mathrm{PT}(\mathrm{s}, \mathrm{di})) \leq \mathrm{Jbound}=\max (\mathrm{J}(\mathrm{e})), \quad \forall \mathrm{e} \in \mathrm{MT}
\end{aligned}
$$

$\operatorname{Pw}(\mathrm{PT}(\mathrm{s}, \mathrm{di})) \leq \mathrm{Pwbound}=\min (\mathrm{Pw}(\mathrm{e})), \forall \mathrm{n} \in \mathrm{MT}$

The following three QoS with the link are defined as follows: e $\in$ ET: The maximum delay variation constraint=JT, The minimum available bandwidth constraint $=\mathrm{BT}$, The minimum power constraint $=\mathrm{PT}$. The path from the sender to receiver has a measured value of jitter ${ }_{\max }$, bandwidth ${ }_{\min }$, and power ${ }_{\min }$ that should be limited by the QoS constraint JT, BT, and PT. The QoS-based routing problem should satisfy QoS constraints given in Eq. 10.The dynamic delay-constrained SP problem is to find a series of multicast tree PT where $\mathrm{T} \in\{0,1, \ldots\}$ through a series of graphs $\mathrm{Gi}[6]$,where $\mathrm{T} \in\{0,1, \ldots\}$, which satisfy the delay constraint, as shown in(12),and have the least path cost, as shown in (13).

$$
\begin{gathered}
\operatorname{Max}\left(D_{T}\right)=\sum_{e \in P_{T}(\mathrm{~s}, \mathrm{di})} \mathrm{d} e \leq \Delta \\
\mathrm{C}(\mathrm{Ti})=\min _{\mathrm{T} \in \mathrm{Gi}}\left\{\sum_{e \in \mathrm{P}(\mathrm{s}, \mathrm{di})} \mathrm{C} e\right\}
\end{gathered}
$$

\section{DESIGN GA FOR DYNAMIC MULTICAST ROUTING PROBLEM}

This section describes the design of the GA for the SP problem. The design of the GA involves several key components: genetic representation, population initialization, fitness function, selection scheme, crossover, and mutation. A routing path consists of a sequence of adjacent nodes in the network. Hence, it is a natural choice to adopt the pathoriented encoding method. For the routing problem, the pathoriented encoding and the path-based crossover and mutation are also very popular [12]

\subsection{Genetic Representation}

String of positive integers uses to encode a routing path that represents ID of nodes, each part of the string represents an arrangement of a node by the gene of the location, the gene of the first location of the source node, and the gene of the last location is the destination node, the routing path length should not greater than the maximum length $|\mathrm{V} 0|$, where $\mathrm{V} 0$ is the set of nodes in the MANET. Chromosomes are encoded under the delay constraint. The encoding processes are repeated to satisfy the delay constraint. The source node is node 1 , the destination node set $D=\{3,9,10\}$.

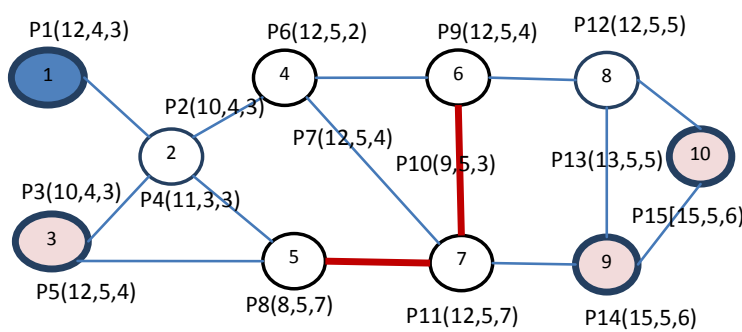

Fig 1: Illustration the multicast tree routing paths of Network Topology Structure

1. The paths that aren't satisfying the delay constraint will 
be eliminated as the follows: $\mathrm{P} 8(5,7), \mathrm{P} 10(6,7)$.

2. The single path between two candidate nodes will selected $\mathrm{P}(1,2,3), \mathrm{P}(9,10)$.

3. The shortest path $=\mathrm{P}(4,7,9)$ will be selected among multiple candidate path as $\mathrm{P}(4,6,8,9)$ and $\mathrm{P}(4,7,9)$.

4. The paths will be collected together to get one path $=\mathrm{P}$ $(1,2,3,4,7,9,10)$ order by path.

5. One line transmission data will be sanded instead of distribution line transmission data.

Unicast provide cost-savings on the construction of Multicastenabled networks while maintaining real-time, low-latency, high-quality video with adequate bandwidth management for all users.

Bandwidth $_{\text {Constraint }}: 100=>\mathrm{B}_{\min }>=10$, Delay ${ }_{\text {Constraint }}: 0=<\mathrm{D}<=5$. Multicast Tree Cost, $C(T)=\min (C(T s), T s \in S(R))$.

Note: bandwidth constraint $\mathrm{B}=10$, delay constraint $\mathrm{D}=5$, generated multicast tree is as shown in Fig.2.

Table 1: Illustration the Candidate Path Set from Source Node to three Destination Node $=\{d 10, d 9, d 3\}$

\begin{tabular}{|c|c|c|c|c|c|c|c|c|}
\hline Destination & \multicolumn{7}{|c|}{ Candidate Path } & Tree \\
\hline 10 & 1 & 2 & 4 & 6 & 8 & 10 & & T1 \\
\hline 10 & 1 & 2 & 4 & 6 & 8 & 9 & 10 & T2 \\
\hline 9 & 1 & 2 & 4 & 6 & 8 & 10 & 9 & T3 \\
\hline 9 & 1 & 2 & 4 & 6 & 8 & 9 & & T4 \\
\hline 9 & 1 & 2 & 4 & 7 & 9 & & & T5 \\
\hline 3 & 1 & 2 & 3 & & & & & T6 \\
\hline 3 & 1 & 2 & 5 & 3 & & & & T7 \\
\hline
\end{tabular}

Both crossover and mutation may produce new chromosomes which represent infeasible solutions, and check whether the multicast trees represented by the new chromosomes are acyclic. If not, the repair function used in will be applied to eliminate the loops. The delay checking is incorporated into both the crossover and mutation operations to guarantee that all the new chromosomes produced satisfy the delay constraint [15].

Table 2: illustration the best path set from Source Node to three Destination nodes used the matrix representation.

\begin{tabular}{|l|l|l|l|l|l|l|l|l|}
\hline Des & \multicolumn{6}{|c|}{ The Best Multicast Tree } & F(B,D,P,J) & Tree \\
\hline 10 & 1 & 2 & 4 & 6 & 8 & 10 & 90 & T1 \\
\hline 9 & 1 & 2 & 4 & 7 & 9 & & 92 & T5 \\
\hline 3 & 1 & 2 & 3 & & & & 96 & T6 \\
\hline
\end{tabular}

\subsection{Population Initialization}

1. The chromosomes have a potential solutions and the initial population $\mathrm{Q}$ is composed of a number of chromosomes.

2. The corresponding routing path is randomly generated for each chromosome in the initial population to enhance the genetic diversity.

3. Randomly searching path from $\mathrm{s}$ to $\mathrm{r}$ and selecting a node $\mathrm{v} 1$ from $\mathrm{N}(\mathrm{s})$, and then select a node $\mathrm{v} 2$ from $\mathrm{N}$ (v1), and it will be repeated until is reached to $r$.
4. All nodes that are already included in the current path are excluded from being selected as the next node to be added into the path.

5. This process will be reiterated $\mathrm{q}$ at times, random path $\mathrm{P}$ $(\mathrm{s}, \mathrm{r})=\{\mathrm{s}, \mathrm{v} 1, \mathrm{v} 2 \ldots \mathrm{rk}\}$. The initial population can be got $\mathrm{Q}=\{\mathrm{Ch} 0, \mathrm{Ch} 1 \ldots$ Chq -1$\}$.

Bandwidth $_{\text {Constraint: }}: 100=>\mathrm{B}_{\min }>=10$,Delay Constraint $_{\text {t }}: 0=<\mathrm{D}<=5$, Multicast Tree Cost, $C(T)=\min (C(T s), T s \in S(R))$.

Note: bandwidth constraint $\mathrm{B}=10$, delay constraint $\mathrm{D}=5$, generated multicast tree is as shown in Fig.3.

\subsection{Fitness Function}

In our algorithms, the Fitness value $\mathrm{F}$ (Chi), used to evaluate the solution quality is the tree cost among a set of candidate solutions, the minimal tree cost will be chosed. Therefore, the Fitness value of chromosome Chi denoted as $\mathrm{F}$ (Chi), is given in Eq. 13.

$F\left(C h_{i}\right)=\left[\sum_{e \in P(s, r)} C e\right]^{-1}$

\subsection{Selection Scheme}

The selection of a chromosome is based on the fitness value for improving the average quality of the population by passing the high-quality chromosomes to the next generation.

Table 3: Illustration the best Path based on fitness (BT, DT, PT, JT) from Source Node to All Destination Nodes.

\begin{tabular}{|c|c|c|c|c|c|c|c|c|c|c|}
\hline Des & \multicolumn{5}{|l}{ Candidate Path } & \multicolumn{2}{l|}{ Fit } & Str & Path \\
\hline 2 & 1 & & & & & & & $\underline{98}$ & S1 & 1 \\
\hline 3 & 1 & 2 & 3 & & & & & $\underline{97}$ & S2 & 2 \\
\hline 3 & 1 & 2 & 5 & 3 & & & & 95 & S3 & 3 \\
\hline 4 & 1 & 2 & 4 & & & & & $\underline{96}$ & S4 & 4 \\
\hline 5 & 1 & 2 & 5 & & & & & $\underline{96}$ & S5 & 5 \\
\hline 5 & 1 & 2 & 3 & 5 & & & & 94 & S6 & 6 \\
\hline 6 & 1 & 2 & 4 & 6 & & & & $\underline{93}$ & S7 & 7 \\
\hline 7 & 1 & 2 & 4 & 7 & & & & 93 & S8 & 8 \\
\hline 8 & 1 & 2 & 4 & 6 & 8 & & & $\underline{90}$ & S9 & 9 \\
\hline 9 & 1 & 2 & 4 & 6 & 8 & 9 & & $\underline{86}$ & S10 & 10 \\
\hline 9 & 1 & 2 & 4 & 6 & 8 & 10 & 9 & 88 & S11 & 11 \\
\hline 10 & 1 & 2 & 4 & 6 & 8 & 10 & & $\underline{82}$ & S12 & 12 \\
\hline 10 & 1 & 2 & 4 & 7 & 8 & 10 & & 81 & S13 & 13 \\
\hline 10 & 1 & 2 & 4 & 6 & 8 & 9 & 10 & 79 & S14 & 14 \\
\hline 10 & 1 & 2 & 4 & 7 & 8 & 9 & 10 & 78 & S15 & 15 \\
\hline
\end{tabular}




\subsection{Crossover and Mutation}

A GA depends on two basic genetic operators - crossover and mutation. Crossover processes the current solutions so as to find better ones. Mutation helps a GA keep away from local optima [2]. The performance of a GA very much depends on them. In this algorithm, the chromosomes are expressed by the path structure, the single-point crossover will be selected to exchange partial chromosomes at appositionally independent crossing sites between two chromosomes [2]. With the crossover probability, each time it select two chromosomes $\mathrm{Ch}_{\mathrm{x}}$ and $\mathrm{Ch}_{\mathrm{y}}$ for crossover with one receiver at least, they should have at least one common node, denoted as $\mathrm{v}$ and randomly selected. In $\mathrm{Ch}_{\mathrm{x}}$ and chromosome, there is a path consisting of two parts: $\mathrm{Ch}_{\mathrm{x}}(\mathrm{s} \rightarrow \mathrm{v})$ and $\mathrm{Ch}_{\mathrm{x}}(\mathrm{v} \rightarrow \mathrm{r})$, and in $\mathrm{Ch}_{\mathrm{y}}$ there is a path consisting of two parts: $\mathrm{h}_{\mathrm{y}}(\mathrm{s} \rightarrow \mathrm{v})$ and $\mathrm{Ch}_{\mathrm{y}}(\mathrm{v} \rightarrow \mathrm{r})$. The crossover operation exchanges the sub paths $\mathrm{Ch}_{\mathrm{x}}(\mathrm{v} \rightarrow \mathrm{r})$ and $\mathrm{Ch}_{\mathrm{y}}(\mathrm{v} \rightarrow \mathrm{r})$, the crossover operation is performed, and the chromosomes will undergo the mutation operation. The mutation will replace all sub path $\mathrm{Ch}_{\mathrm{x}}(\mathrm{v} \rightarrow \mathrm{r})$ with new random all sub path $\mathrm{Ch}_{\mathrm{y}}(\mathrm{v} \rightarrow \mathrm{r})$. The crossover and mutation operations are including the delay checking to guarantee that all new chromosomes produced satisfy the delay constraint, Multi Objective Constraints < BT, DT, PT, JT > where BT -Bandwidth Tree; DT- Delay Tree; PTPowerTree; JT-Jitter Tree.

\begin{tabular}{|l|l|l|}
\hline Index & $\mathrm{Ni}$ & $\mathrm{Si}=\mathrm{Ti}$ \\
\hline 1 & 1 & 0 \\
\hline 2 & 2 & 1 \\
\hline 3 & 3 & 2 \\
\hline 4 & 4 & 2 \\
\hline 5 & 6 & 4 \\
\hline 6 & 8 & 6 \\
\hline 7 & 9 & 8 \\
\hline 8 & 10 & 8 \\
\hline 9 & 7 & 4 \\
\hline 10 & 5 & 2 \\
\hline
\end{tabular}

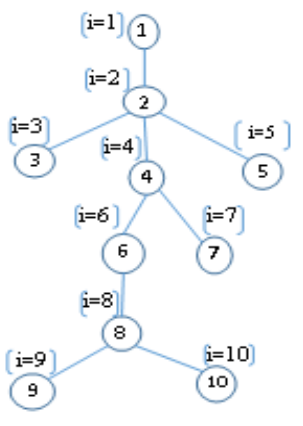

Fig 2: Illustration the multicast tree routing paths of Network Topology Structure satisfying QoS constraints.

The main process in GA is to define an encoding representation and break the process down into six steps:

Step 1: The encoding approach will be simple for crossover and mutation operation. In this paper, the sequence and the topology encoding method which maps candidate solutions as an integer string are explained.

Step 2: The chromosome represents a feasible solution based on its fitness function. The maximum value of the fitness value will be selected to survive and regenerate.

Step 3: different selection criteria are used to take the best individuals from new generation genomes as chromosomes and then applied crossover and mutation among individuals in the population to generate new individuals.

Step 4: All trees should be indexed to be encoded with sequence and topology encoding based on number of nodes $(\mathrm{Ni}, \mathrm{Si}, \mathrm{Ti})$ as string where $\mathrm{Ni}$ is the number of nodes of the indexed tree and $\mathrm{Si}$ is a sequence encoding. The string $\mathrm{Ti}$ is used to encode the topology of the tree.

Step 5: The encoding algorithm used to represent the multicast tree into the sequence and topology encoding used three arrays $\mathrm{Ni}, \mathrm{Si}, \mathrm{Ti}$ where $\mathrm{i}$ as the position index. Where the root of multicast tree $\mathrm{T}$ into the first element of string $\mathrm{T}$ [1] $=\mathrm{S}[1]=\mathrm{T}[1]$ represents the position index of the source node for host $\mathrm{Si}$ where $\mathrm{i}=1$. The root host starts from the first Node as source and its tree has no predecessor $\mathrm{T}$ [1] $=0$. All other nodes, put the node number at index I of the tree into a string.

Step 6: The procedure of decoding to convert sequence and topology code into its corresponding tree is in the following:

First, set $\mathrm{S}[\mathrm{I}]$, where $\mathrm{i}=1$, and the root of multicast tree $\mathrm{T}$. For $\mathrm{i}=2$ to $\mathrm{n}$, set a link $\mathrm{S}[\mathrm{I}]$ to $\mathrm{S}[\mathrm{n}]$. Fig.3 (a) andFig.4 (a) have the sequence and topology codes shown in Fig. 3 (b) and Fig. 4 (b). , string $\mathrm{s} 1=\{1,2,3,4,6,8,9,10,7,5\}$ and $\mathrm{t} 1=$ $\{0,1,2,2,4,5,6,6,4,2\}$, and string $s 2=\{1,9,8,7,3,5,6,4,2\}$ and $\mathrm{t} 2=\{0,1,2,2,1,5,6,6,1\}$. T1 is used to encode the topology of the tree. The interior nodes of the multicast tree are selected from strings $\mathrm{S} 1$ by the order of the index numbers, showing in strings $\mathrm{Si}$.

Table 4: Illustration the sequence multicast tree with indexed nodes and encoding of Network Topology Structure.

\begin{tabular}{|c|c|c|c|c|}
\hline S14 & $\mathrm{S} 12$ & S13 & & $\mathrm{S} 15$ \\
\hline 1 & 1 & 1 & $4-$ & 1 \\
\hline 2 & 2 & 2 & $1 \rightarrow-$ & 2 \\
\hline$\underline{4}$ & 4 & 4 & -1 & $\underline{4}$ \\
\hline 6 & 6 & $\underline{6}$ & & 7 \\
\hline 8 & 8 & 7 & & 8 \\
\hline 9 & 10 & 10 & & 9 \\
\hline 10 & & & & 10 \\
\hline
\end{tabular}

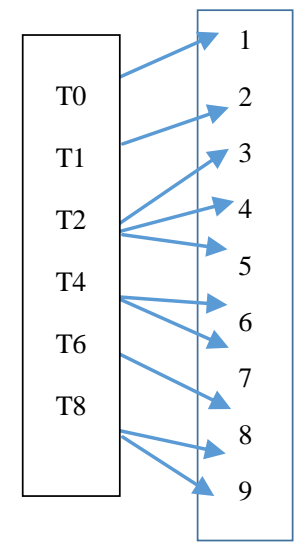

Fig 3: The crossover and mutation process to generate new chromosome from Source Node No1 to Destination Nodes.

Table 6: Illustration the best general path and minimum sequence delay used the matrix representation

\begin{tabular}{|l|l|l|l|l|l|l|l|l|l|l|}
\hline & N1 & N2 & N3 & N4 & N5 & N6 & N7 & N8 & N9 & N10 \\
\hline T0 & d1 & & & & & & & & & \\
\hline T1 & & d2 & & & & & & & & \\
\hline T2 & & & d3 & d4 & d5 & & & & & \\
\hline T4 & & & & & & d6 & d7 & & & \\
\hline T6 & & & & & & & & d8 & & \\
\hline T8 & & & & & & & & & d9 & d10 \\
\hline Fit & 100 & 98 & 97 & 96 & 96 & 93 & 93 & 90 & 86 & 82 \\
\hline
\end{tabular}


Delay $\mathrm{F}(\mathrm{D})=$ Delay $\mathrm{D}\left(\mathrm{d}_{\mathrm{Ti} \in \mathrm{T}}\right)$

The Total Delay Sequence of the general path $\mathrm{P}_{\mathrm{T}}(\mathrm{s}, \mathrm{D})$ is

$f(T)=T i \in \mathrm{T}$

The Total Tree Sequence of the general path PT (s, D) is:

$f(D)=\mathrm{d}(T i \in \mathrm{T})$

The Total Bandwidth Sequence of the general path $\mathrm{P}_{\mathrm{T}}(\mathrm{s}, \mathrm{D})$ is

$f(B)=(T i \in \mathrm{T})$

The Total Cost Sequence of the general tree as the cost sum of all trees.

$f(C)=(T i \in \mathrm{T})$

\section{ENERGY EFFICIENT AND MULTICASTING}

The energy efficiency is the main constraint packet error probability since a node is battery powered, the link must be considered energy-critical and calculate the energy cost of each link to determine expected energy from node to another node for transmitting a packet [21], and the reliability (E) is used to construct the energy efficient trees. $\mathrm{E}=\mathrm{E} /$ (1$\mathrm{Pij}$ ), where $\mathrm{Pij}$ is the probability packet error probability between node $I$ and $\mathrm{j}$ and $1 /(1-\mathrm{Pij})$ is the expected number of retransmissions required from node $i$ to $j$, The data packet transfer through more than one link to a destination, leads to reduce the energy and multicast efficiency, and also the multicast routing does not support an energy-conserving to increase the lifetime of the network, it is important to find energy-conserving schemes and protocols that optimize the use of battery power, calculate the energy level of a node $\mathrm{N}$ $\mathrm{i} \rightarrow, \mathrm{j}, \mathrm{N} \in \mathrm{V}(\mathrm{G}) . \mathrm{Ei} \rightarrow, \mathrm{j}$ is denoted the residual energy of $\mathrm{N}$ node, and Emax denote he full energy divided into all nodes $\mathrm{L}$ , $\mathrm{L}$ is a parameter of network, where a link of node $\mathrm{Li} \rightarrow, \mathrm{j} \in \mathrm{E}$ $(\mathrm{G})$, the energy level of the link ( ERL) as follows.

$$
\text { ERL L* En/E } \mathrm{E}_{\max }
$$

The energy-efficient multicasting protocols (E2MRP) for MANETs operates in two phases. The first phase uses a heuristic called minimum energy consumed per packet (MECP), the received power at a node is directly proportional to $d^{a}$ where $d$ is the distance from sender to receiver and $a$ is the constant , $\mathrm{Pij}$ is the power required to transmit a packet from node $i$ to $j$, Dij is the distance from $i$ to $j, C P$ is the power cost for a route from source $\mathrm{i}$ to destination $\mathrm{n}$.

$C P=\sum_{i=1}^{n} P i, i+1=\sum_{i=1}^{n} d 2(N i, N j)$

Where I varies from 1 to $\mathrm{N}$, the $\mathrm{CP}$ with the lowest value is selected for the route, the main idea is to minimize the total end-to-end power consumed in sending a packet from the source to its final destination. And the second phase uses a heuristic called minimum maximum node cost (MMNC).

$\operatorname{Ci}(\operatorname{Vi}(t))=Y / \operatorname{Vi}(t)$

Where $\mathrm{Y}$ is constant, $\mathrm{Vi}(\mathrm{t})$ is the energy consumed by node $\mathrm{i}$ until time t. $\mathrm{CP}=\max (\mathrm{Ci}(\mathrm{Vi}(\mathrm{t}))$ and the route with Minimum $\mathrm{CP}$ is selected ,operation protocol switches between MECP and MMNC, Mesh is first formed using MECP and a Cost Function Switcher is set (CFS), Once CFS times out, the system switch to MMNC and when the timer Expires, Switches to MECP, this heuristic gives more importance to the power available at nodes along the route.

\section{SIMULATION RESULTS}

The QoS multicast routing protocol is simulated in MANET by using Ns2 simulator at networking research, Ns2 provides substantial support for simulation of TCP, routing, and multicast protocols over wireless networks Ns2 to be the platform, MOGAs and RIMOGAs are implemented for the DSPRP, and their performance are evaluated in a continuously changing wireless network [18], The network topology is generated in the Dynamic Test Environments by specifying a rectangular region of $1000 \mathrm{~m}$ by $1000 \mathrm{~m}$. and then generate 100 nodes, and the position of each node is randomly selected within the square area, with node speed between $(0-$ 20)Mps If the distance between two nodes within the scope of broadcasting and into the radio transmission range, a link will be a4ded to connect between them, so that the cost and the delay of the link are randomly specified into the corresponding ranges, and maximum delay $=40$ seconds , minimum bandwidth $=10 \mathrm{mbps}$, the generated topology will be tested is connected or not. If not connected, the previous process will be repeated to be connected topology and generated. the chromosomes fitness will evaluate for each generation. Fitness is estimated based on the multi-objective genetic algorithms MOGAs in most cases of optimization fitness functions. , a better chromosome has larger fitness value. The fitness value of each chromosome is defined as Eqs. 13. And compare extended tree encoding and ST encoding g, Fig.4 illustrate the experiment results of MOGAs and the fitness values in various generations, after running 1000 generations The results are obtained, the extended tree encoding has a higher fitness value .

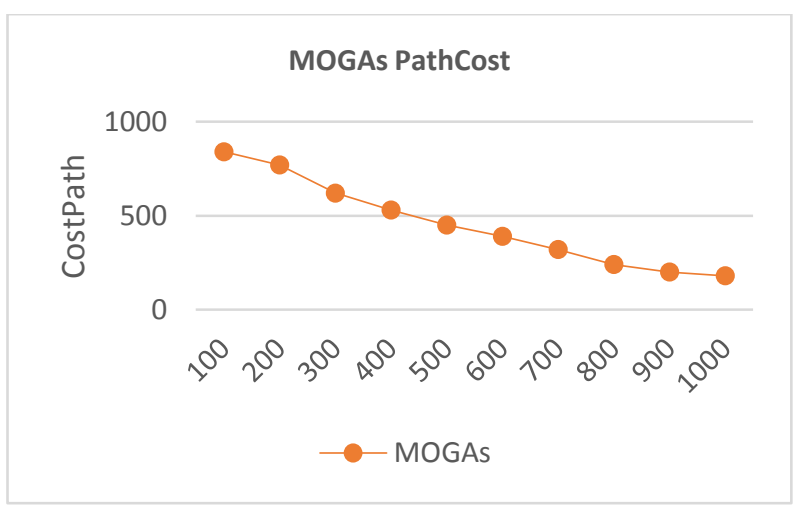

Fig 4: Comparison of the solution quality of MOGAs for The fitness values in various generations 0 -1000.

In the Figure 5. the effect of the population size investigated on the performance of RIMOGAs and determine a population size to be ensured a specified quality of solutions. Shows that in the environment with a high change severity with RIMOGAs, all feasible individuals are replaced by random immigrants [16], RIMOGAs applied to run over topology. The generation set to $100,200,300 \ldots 1000$ respectively. The population size was varied $10,20,30,40$ to check if the population size can be determined for this problem, and there are 10 topologies, the first 1000 generations checked to represent the figures. Figure 1 shows the results over topology and Compare the solution quality of RIMOGAs and MOGAs. 


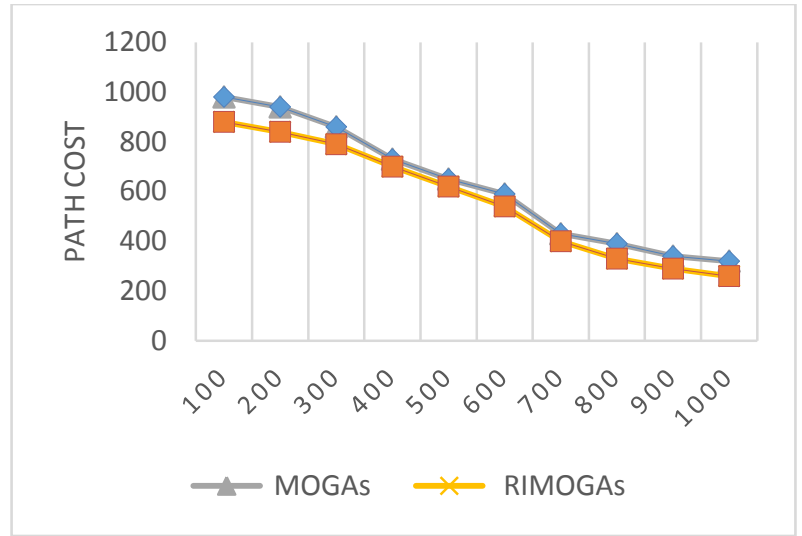

Fig 5: Comparison of the solution quality of HIMOGAs and RIMOGAs with different change intervals generation 0-1000

Figure 6 shows that in the environment with a high change severity, the all infeasible individuals are repaired to become feasible and the EIMOGA is re-selected, and performs very well after 10 generations to get the overall best solution. The reason is that in the highly dynamic environment and after a change occurs, the proposed repair method can reserve the useful components of the EIMOGAs and repair the broken part with the least added cost. The new immigrants generated by repairing the EIMOGAs can quickly adapt to the severe changes, the have discussed under the general dynamics model, EIMOGAs gets a less diversity to the population, compared to RIMOGAs and MOGAs, it can conclude that these dynamic GAs respond to the environmental changes in a reasonable speed and perform well.

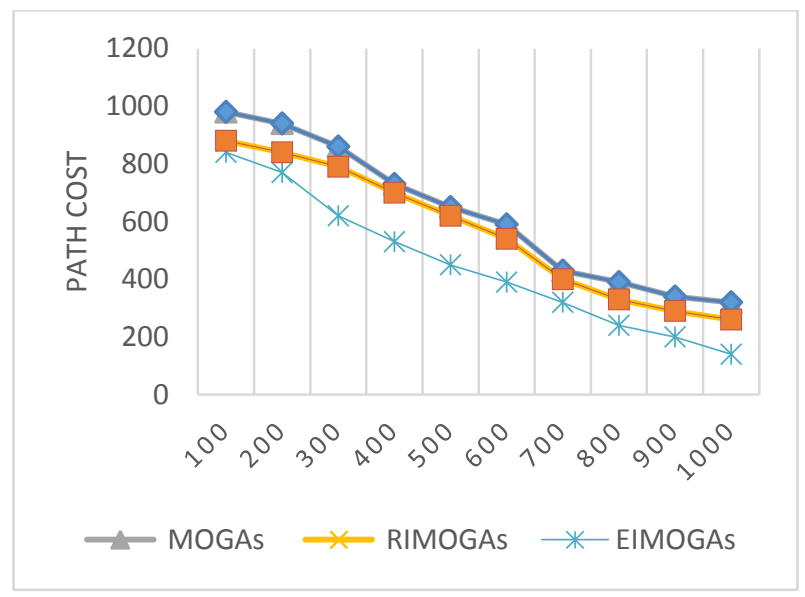

Fig 6: Comparison of the solution quality of MOGAs, RIMOGAs, EIMOGAs with different change intervals generation 0-1000.

Figures 7 shows that on the average HIMOGAs achieves the best performance at the population size of 90 . When the population size is increased to 100 , the algorithm performance not change on most of the time, it conclude that 90 is the best choice for the population size for our problem. They can also be seen that many times when a change occurs, the algorithms are not aff ected. The reason is that the topology changes may not always aff ect the current population, especially the optimal individual in the population. For example, if the nodes that are scheduled to sleep or wake up in one change are not on the tree represented by the optimal individual, the optimal individual has a very high probability to stay in the population unaff ected. HIMOGAs can quickly adapt to the environmental changes and get the best solution among these three MOGAs, RIMOGAs and EIMOGAs performs the best in the environment with a low change severity and gets the advantages of RIMOGAs and EIMOGAs.

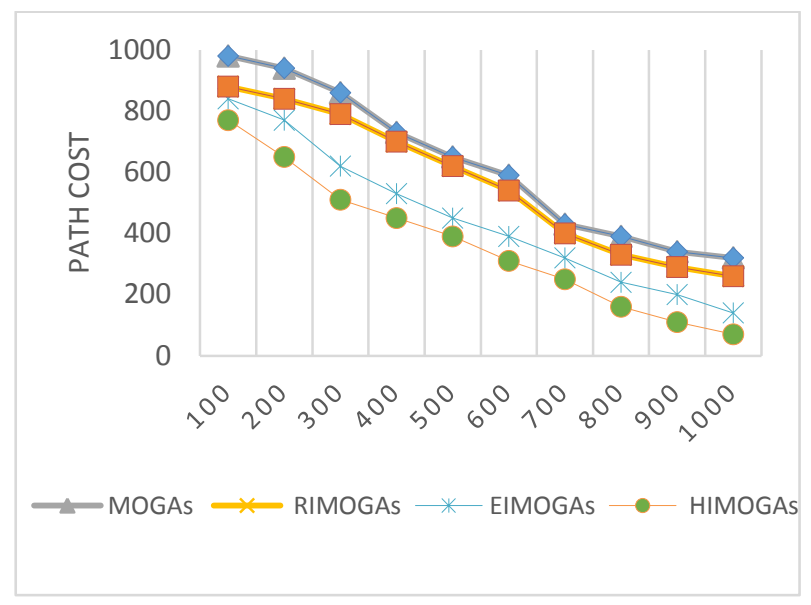

Fig 7: Comparison of the solution quality of SMOGA, RIMOGAs EIMOGAs and HIMOGAs with different change interval generation

In the Figure 8.1 and 8.2, HIMOGAs is simulated to develop a multicast transmission with load balancing scheme to be minimized and the maximized the link utilization. The total bandwidth consumption, the total end-to-end delay, maximum residual energy for each link and also discover the network lifetime performance with different population size (pop_size $=100$,pop_size $=200$ ). In the experiments supposed the energy critical ratio $(E C R=25), E C R$ is percent of the links in the neighborhood with the energy-critical value, to calculate the energy level of a node, the full energy is divided into all nodes to be as a parameter of network and energy level of the link, they set $25 \%$ of links to be energy critical $(\mathrm{ECR}=25)$, and $50 \%$ of links with random values generation of energy (from 10 to 50), the following four QoS with the link are defined : The maximum delay constraint $=40$ second, the minimum available bandwidth constraint $=10 \mathrm{mbps}$, and the minimum power constraint $=25 \mathrm{w}$. This results from the fact that the proposed algorithm optimize the use of network resources. These results show that, when multiple objectives are optimized [18], while some objectives are improved, we can see that, the proposed algorithm (HIMOGAs) behaves in a satisfactory way, minimizing some variables, the results of the quality of solution and the response speed and demonstrate that our algorithm can adapt to the environmental changes well and achieve better solutions after each change. Therefore, they are good techniques for working with Multi-Objectives for dynamic environment problems and satisfy the multiobjective constraints.

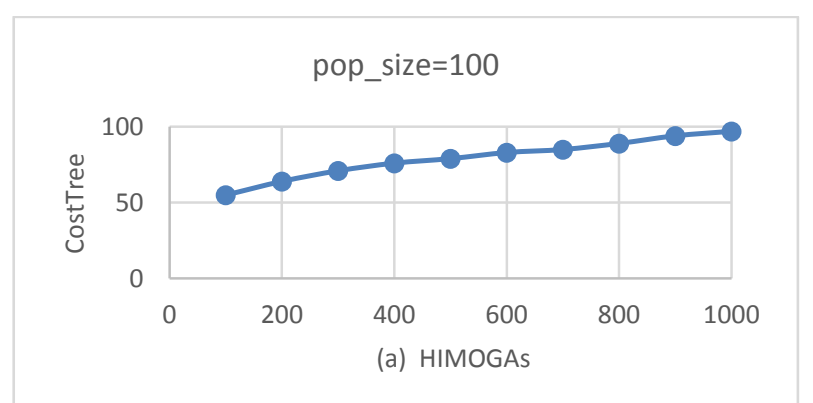

Fig 8.1: The results of the quality of solution and the response speed for HIMOGAs, with population size $=100$. 


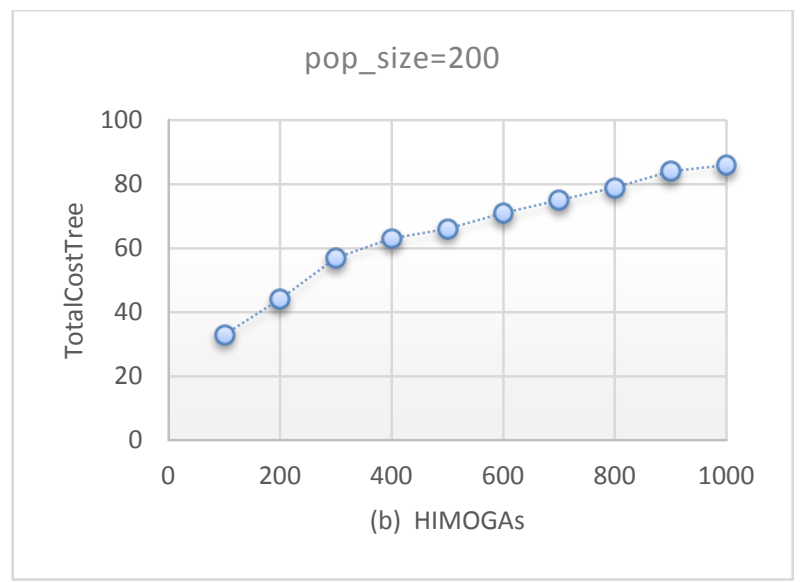

Fig 8.2: The results of the quality of solution and the response speed for HIMOGAs with population size $=200$.

\section{CONCLUSIONS AND FUTURE WORK}

This paper proposes a Hybrid Immigrants Scheme [17] and Multi-Objectives genetic algorithms for the quantity of services, multicast routing method, this proposal has used the available resources and minimum computation time in a dynamic environment to select the appropriate, mutation, and population size, the HIMOGAs improves and optimizes routes. The experiment results indicate its better performance compared to another algorithm. Our proposed algorithm has many advantages and features: a) Multi-Constrained used available limited resources to control transmission packages that effectively decreases the search space and eliminates the probability of selecting undesirable nodes and links. b) For the sequence and topology encoding, they can use method that provides a tree-based structure coding scheme to simplify the coding and decoding process. c) Improve the fitness function that allows the proposed HIMOGAs to be applied to other multicast problems to compute multicast trees with minimum delay time, maximum bandwidth and higher battery energy. d) A couple of chromosomes, are a set of one paternal trees that pair up with each other, they copies have the same genes in the same location where they provide points along each chromosome, which enable a pair of chromosomes to align correctly with each other and it is best suited for mixing genes [1]. f) We propose an energy efficient localized topology control algorithm, the node is required to maintain its one-hop neighborhood topology. In order to achieve long network lifetime and mutation operation allow the core node with a lower energy to be replaced by another node with a greater energy. In the future research, there are several issues as follows: a) improve various route discovery and recovery mechanisms are more robust with various network changing and failures. b) How making the cost function more precisely. c) Improve the design of routing protocols and calculate the multiple metrics for each received and rebroadcast connection-request.

\section{REFERENCES}

[1] F. Oppacher and M. Weinberg, "The shifting balance genetic algorithm: Improving the GA in a dynamic environment," Proc. 1999 Genet. Evol. Comput. Conf., vol. 1, pp. 504-510.

[2] C. W. Ahn, R. S. Ramakrishna, C. G. Kang, and I. C. Choi, "Shortest path routing algorithm using Hopfield neural network," Electron. Lett, vol. 37, no. 19, pp. 1176-1178, Sep. 2001.
[3] C. W. Ahn and R. S. Ramakrishna, "A genetic algorithm for shortest path routing problem and the sizing of populations," IEEE Trans. Evol. Comput., vol. 6, no. 6, pp. 566-579, Dec. 2002.

[4] J.Branke, "Memory enhanced evolutionary algorithms for changing optimization problems," inProc.1999Congr. Evol. Comput, pp. 1875-1882.

[5] J. Branke, Evolutionary Optimization in Dynamic Environments. Norwell, MA: Kluwer, 2002.

[6] Parsa, M., Zhu, Q., Garcia-Luna-Aceves, J. (1998). An Iterative Algorithm for Delay-constrained Minimum-cost Multicasting. IEEE/ACM Trans. Netw., 6, 461-474.

[7] Jain, K., Padhye, J., Padmanabhan, V., and Qiu, L. (2003). Impact of Interference on Multi-hop Wireless Network Performance. Proc. MobiCom 2003, 66-80.

[8] B. L. Sun, S.C.Pi, C. GUI, et al., Multiple constraints QoS multicast routing optimization algorithm in MANET based on GA, Progress in Natural Science 18 (3) (2008)331-336.

[9] J. Branke, T. Kaußler, C. Schmidt, and H. Schmeck, “A multi population approach to dynamic optimization problems,"inProc. 4thInt.Conf.Adaptive Comput. Des. Manuf., 2000, pp. 299-308.

[10] X. Yu, K. Tang, and X. Yao, “An immigrants scheme based on environmental information for genetic algorithms in changing environments," in Proc. 2008 Congr. Evol. Comput., pp. 1141-1147.

[11] M. Parsa, Q. Zhu, and J. Garcia-Luna Aceves, "An iterative algorithm for delay-constrained minimum-cost multicasting," IEEE/ACMTrans. Netw., vol. 6, no. 4, pp. 461-474, Aug. 1998.

[12] R. Tinos and S. Yang, "A self-organizing random immigrants genetic algorithm for dynamic optimization problems," Genet. Program. Evol. Mach., vol. 8, no. 3, pp. 255-286, Sep. 2007.

[13] C. -K. Toh, Ad Hoc Mobile Wireless Networks: Protocols and Systems. Englewood Cliffs, NJ: PrenticeHall, 2002.

[14] S. Yang, "Population-based incremental learning with memory scheme for changing environments," in Proc. 2005 Genet. Evol. Comput. Conf., vol. 1, pp. 711-718.

[15] S. Yang, "Genetic algorithms with elitism-based immigrants for changing optimization problems," in Proc. EvoWorkshops 2007: Appl. Evol. Comput. (Lecture Notes in Computer Science), vol. 4448, pp. 627-636.

[16] S. Yang,"Genetic algorithms with memory-and elitismbased immigrants in dynamic environments," Evol. Comput., vol. 16, no. 3, pp. 385-416, Sep. 2008.

[17] S. Yang and R. Tinos, "A hybrid immigrants scheme for genetic algorithms in dynamic environments," Int. J. Autom. Comput., vol. 4, no. 3, pp. 243-254, Jul. 2007.

[18] Baolin Sun and Layuan Li, Multiple Constraints Based QoS Multicast Routing: Model and Algorithms, Journal of Systems Engineering and Electronics, Vol. 15, No. 4, 2004.

[19] V. Rodoplu and T. H. Meng, "Minimum energy mobile wireless networks," IEEE. J. Sel. Areas Commun., vol. 
17, no. 8, pp. 1205-1220, 2008.

[20] H. Liu, B. Zhang, J. Zheng, and H. T. Mouftah, "An energy-efficient localized topology control algorithm for wireless ad hoc and sensor networks," Int. J. Commun. Syst., vol. 21, no. 11, pp. 1205-1220, 2008.

[21] Wang, X., Cao, J., Cheng, H., and Huang, M. (2006). QoS Multicast Routing for Multimedia Group Communications Using Intelligent Computational Methods. Comput. Comm., 29, 2217-2229.

[22] S. -Y. Tseng, Y. -M. Huang, C. -C. Lin, Genetic algorithm for delay and degree-constrained multimedia broadcasting on overlay networks, Computer Communications 29 (17) (2006)3625-3632.

[23] Das and C. Martel,"Stochastic shortest path with unlimited hops,” Inf. Process. Lett. vol. 109, no. 5, pp. 290-295, 2009

[24] Yen, Yun-Sheng, et al. "Flooding-limited and multiconstrained QoS multicast routing based on the genetic algorithm for MANETs." Mathematical and Computer Modelling 53.11 (2011): 2238-2250. 